\title{
Entanglement measures with asymptotic weak-monotonicity as lower (upper) bound for the entanglement of cost (distillation)
}

\author{
Won-Young Hwang*, and Keiji Matsumotoẗ \\ IMAI Quantum Computation and Information Project, ERATO, Japan Science Technology Cooperation, Daini Hongo White \\ Bldg. 201, 5-28-3, Hongo, Bunkyo-ku, Tokyo 133-0033, Japan
}

\begin{abstract}
We propose entanglement measures with asymptotic weakmonotonicity. We show that a normalized form of entanglement measures with the asymptotic weak-monotonicity are lower (upper) bound for the entanglement of cost (distillation).
\end{abstract}

03.67.-a

It is quantum entanglement that led to the controversy over Einstein-Podolsky-Rosen experiment [1] and the non-local nature of quantum mechanics [2].

The entanglement is the key ingredient in quantum information processing. The speedup in quantum computation [3] is obtained through the parallel quantum operations on massively superposed states which are in general entangled. In particular, in quantum communications such as the quantum teleportation [4] the entanglement is valuable resource that should not be wasted, since entanglements can only be obtained by (costly) non-local operations. For better understanding and manipulation of entangled states, we need to classify them as well as possible. Quantification of the entanglementdegree namely the measure of entanglement is, thus, the central issue in quantum information theory [5 7].

A pair of important measures of entanglement, that is, the entanglement of cost $E_{C}$ and entanglement of distillation $E_{D}$ were introduced in a pioneering work by Bennett et al. [5]. Recently the relation between the entanglement of cost $E_{C}$ and the entanglement of formation $E_{f}$ [5] has been clarified [10]. That is, $E_{C}(\rho)=\lim _{n \rightarrow \infty} E_{f}\left(\rho^{\otimes n}\right) / n$. (Here $\rho$ denotes a mixed state.) The pair of measures of entanglement have been shown to be the limits for other entanglement measures satisfying certain conditions [8,9]. In other words, any entanglement measure satisfing the conditions is the lower (upper) bound of the entanglement of cost $E_{C}$ (entanglement of distillation $E_{D}$ ). One of the conditions for the entanglement measures is monotonicity [7] that entanglement measures cannot be increased by any local quantum operations with classical communications (LOCCs).

On the other hand, it has been shown that there is irreversibility in the asymptotic manipulations of entanglement [11]. That is, $E_{D}(\rho)<E_{C}(\rho)$ for a class of states $\rho$ for which $E_{D}(\rho)=0$. However, due to possible non-additivity it is still formidable task to calculate even bounds for the $E_{C}$ and $E_{D}$ except for a few cases [11 15$]$. Thus it is not yet clear whether $E_{C}(\rho)$ is strictly greater than $E_{D}(\rho)$ in general.
In this paper, we consider a relaxed and thus conceptually simple monotonicity requirement, namely asymptotic weak-monotonicity. We show that certain reparameterized entanglement measures satisfying the relaxed monotonicity are the lower (upper) bound for the entanglement of cost $E_{C}$ (entanglement of distillation $\left.E_{D}\right)$. These bounds are different from those in Ref. [8, 9]. It is notoriously difficult to calculate the asymptotic measures of entanglement $E_{C}, E_{D}$, or even bounds to them as noted above. Thus it is worthwhile for us to give clues for the bounds to the asymptotic measures of entanglement, namely the entanglement measures with asymptotic weak-monotonicity.

Now let us review basic conditions for the entanglement measures [16]. (a) Non-negativity: $E(\rho) \geq 0$. (b) Vanishing on separable states: $E(\rho)=0$ if $\rho$ is separable. In addition to these, we require monotonicity conditions [16. Let LOCCs transform an initial state $\rho_{\text {initial }}$ into a final state $\rho_{\text {final }}$. The weak-monotonicity is that the entanglement degree does not increase with LOCCs. (c.1) The weak-monotonicity [17]: $E\left(\rho_{\text {initial }}\right) \geq E\left(\rho_{\text {final }}\right)$. Let us consider the case where LOCCs transforms an initial state $\rho_{\text {initial }}$ into an ensemble $\left\{p_{j}, \rho_{\text {final }}^{j}\right\}$. Here $j$ is positive integer and $p_{j}$ is the probability that $\rho_{\text {final }}^{j}$ will outcome. The strong-monotoncity says that the expectation value of the entanglement degree does not increase with LOCCs. (c.2) The strong monotonicity: $E\left(\rho_{\text {initial }}\right) \geq \sum_{j} p^{j} E\left(\rho_{\text {final }}^{j}\right)$. Let us introduce the asymptotic weak-monotonicity. Consider the asymptotic transformation. Let us consider transformation of an initial state $\rho_{\text {initial }}^{\otimes n_{i}}$ to a final state $\rho_{\text {final }}^{\otimes n_{f}}$. ( $n$ 's are positive integers.) Here it does not matter whether the initial state is transformed to different state during the intermediate processings. Finally, however, the state is reduced to identical copies of a single state, $\rho_{\text {final }}^{\otimes n_{f}}$. We say that a state $\rho_{\text {initial }}$ can asymtotically be transformed to a state $\rho_{\text {final }}$, if we can achieve a transformation $\rho_{\text {initial }}^{\otimes n_{i}} \Rightarrow \rho_{\text {final }}^{\otimes n_{f}}$ at least apporoximately by LOCCs with a condition $n_{i} \leq n_{f}$. More precise definition is the following.

Definition 1: We say that a state $\rho_{\text {initial }}$ can asymptotically be transformed to a state $\rho_{\text {final }}$ with LOCCs, if the followings are satisfied. For any $\epsilon, \eta$, and $\delta>0$, there exist an $M$ such that for any $n_{i} \geq M$ we can transform a state $\rho_{\text {initial }}^{\otimes n_{i}}$ with LOCCs to a state $\xi$, with the fidelity $F\left(\xi, \rho_{\text {final }}^{\otimes n_{f}(1-\delta)}\right) \geq 1-\epsilon$ and with the success probability $\mathrm{P} \geq 1-\eta$. Here $n_{i} \leq n_{f}$ and the $F\left(\rho, \rho^{\prime}\right)=\operatorname{tr} \sqrt{\sqrt{\rho} \rho^{\prime} \sqrt{\rho}}$ is the Uhlmann fidelity 18,19 . 
Asymptotic weak-monotonicity condition (c.3) If an initial state $\rho_{\text {initial }}$ can be asymptotically transformed to a final state $\rho_{\text {final }}$ by LOCCs, then $E\left(\rho_{\text {initial }}\right) \geq E\left(\rho_{\text {final }}\right)$.

In the asymptotic weak-monotonicity (c.3), exact transformation is not required: Even though we cannot exactly transform an initial state to a desired final state, we require that $E\left(\rho_{\text {initial }}\right) \geq E\left(\rho_{\text {final }}\right)$ as long as we can asymptotically achieve the transformation.

Let us disscuss on the difference between the entanglement measure with asymptotic weak-monotonicity and that of strong-monotonicity [7]. Consider the case where entanglement measures with strong-monotonicity are transformed by a monotonic function $m$. That is, consider a re-parameterized function $E^{\prime}(\rho)=m(E(\rho))$. Here we assume that the re-parameterized entanglement measure $E^{\prime}(\rho)$ satisfy the two obvious conditions (a) and (b). The re-parameterized function $E^{\prime}(\rho)$ is no longer the entanglement measure with strong-monotonicity in general: the entanglement measure involves with processes where the number of distinguishable states varies. For example, measurements can transform an initial state into different states. In mixing processes, different states are transformed to a single state. In the both cases, strongmonotonicity requires that the expectation value of entanglement cannot increase. However, it is easy to see that the re-parameterization does not preserve strongmonotonicity in general with respect to the processes where the number of the states varies. However, it is clear that the asymptotic weak-monotonicity is preserved upon the re-parameterization, since we are considering the one-dimensional orderings in this case.

Proposition 1: The asymptotic weak-monotonicity is preserved upon the re-parameterization. Namely, if $E(\rho)$ is an entanglement measure with asymptotic weakmonotonicity and $E^{\prime}(\rho)=m(E(\rho))$ where $m$ is a monotonic function, then $E^{\prime}(\rho)$ is also an entanglement measure with asymptotic weak-monotonicity.

Proposition 2: All entanglement measures with asymptotic weak-monotonicity gives rise to the same orderings for pure states.

Proof: Let us assume two entanglement measures $E_{i}$ $(i=A, B)$ with asymptotic weak-monotonicity give rise to different orderings for two pure states $\rho$ and $\rho^{\prime}$. The fact that the order is reversed in dependence on entanglement measures obviously means that entanglementdegree of $\rho$ is less than that of $\rho^{\prime}$ in one of the two measures and vice versa in the other. That is, we have either

$$
E_{A}(\rho)>E_{A}\left(\rho^{\prime}\right) \text { and } E_{B}(\rho)<E_{B}\left(\rho^{\prime}\right),
$$

or

$$
E_{A}(\rho)<E_{A}\left(\rho^{\prime}\right) \text { and } E_{B}(\rho)>E_{B}\left(\rho^{\prime}\right) .
$$

Due to the asymptotic weak-monotonicity condition (c.3) and Eqs. (1) and (2), the state $\rho$ can neither be asymptotically transformed to the state $\rho^{\prime}$ nor $\rho^{\prime}$ to $\rho$. That is, the two states $\rho$ and $\rho^{\prime}$ are (asymptotically) incomparable 20 22]. However, for the two pure states $\rho$ and $\rho^{\prime}$, we can achieve the asymptotic transformation of either $\rho^{\otimes n} \Rightarrow\left(\rho^{\prime}\right)^{\otimes n}$ or $\rho^{\otimes n} \Leftarrow\left(\rho^{\prime}\right)^{\otimes n}$, by entanglement concentration and dilution [23]. This is in contradiction with the former statement.

The Proposition 1 says that we can generate numerous entanglement measures with asymptotic weakmonotonicity from a single one. On the other hand, the unique entanglement measure for pure states is also an entanglement measure with asymptotic weakmonotonicity for pure states. Here the unique measure for a pure state is the followings. $E_{p}(|\Psi\rangle\langle\Psi|)=$ $S\left(\operatorname{Tr}_{B}|\Psi\rangle\langle\Psi|\right)$, where $S(\rho)=\operatorname{Tr}\left(-\rho \log _{2} \rho\right)$ and $B$ denotes the latter one of the two parties Alice and Bob [24,7]. Thus by the proposition 2 for pure states the ordering of the entanglement measures with asymptotic weak-monotonicity is the same as that of the unique measure of entanglement. Therefore, we can fix the freedom of entanglement measure $E$ with asymptotic weakmonotonicity involved with the Proposition 1 by the following condition.

Normalization condition: For any pure state $\rho$, $E(\rho)$ is the same as the unique entanglement measure $E_{p}(\rho)$ for pure state 24.7].

Although the normalization condition is involved with entanglement-degrees only for pure states, it is also fixing those for mixed states since entanglement-degrees of pure states are continously distributed 25]. We denote the entanglement measure with asymptotic weak-monotonicity satisfying the normalization condition as $\tilde{E}$.

Proposition 3: The normalized entanglement measure with asymptotic weak-monotonicity $\tilde{E}(\rho)$ is the lower bound for the entanglement of cost $E_{C}(\rho)$.

Proof: Assume that there exists a state $\rho$ such that $E_{C}(\rho)<a<\tilde{E}(\rho)$. Then we choose a pure state such that $E_{p}(|\Psi\rangle\langle\Psi|)=a$. This means that we can asymptotically distill $m a$ numbers of the maximally entangled states $\left(\left|\phi^{+}\right\rangle\left\langle\phi^{+}\right|\right)^{\otimes m a}$ from the $m$ copies of the state $|\Psi\rangle\langle\Psi|$. (Here $\left|\phi^{+}\right\rangle=(1 / \sqrt{2})(|00\rangle+|11\rangle)$.) Then some of the ma numbers of the maximally entangled states can be asymptotically transformed to $\rho^{\otimes m}$ [21], since $m E_{C}(\rho)<m a$. What we have done is the asymptotic transformation $|\Psi\rangle\langle\Psi| \Rightarrow \rho$. By the definition 1, we have $\tilde{E}(|\Psi\rangle\langle\Psi|) \geq \tilde{E}(\rho)$. (Note that the continuity condition is not additionally necessary here while it is in the case of Ref. [8,9]. The asymptotic weak-monotonicity is defined such that the continuity condition is not necessary.) By the normalization condition, we have $\tilde{E}(|\Psi\rangle\langle\Psi|)=a$. Thus we have $\tilde{E}(|\Psi\rangle\langle\Psi|)<\tilde{E}(\rho)$, which contradicts above obtained inequality.

We can get a proposition for the entanglement of distillation $E_{D}(\rho)$ in a similar way.

Proposition 4: The normalized entanglement measure with asymptotic weak-monotonicity $\tilde{E}(\rho)$ is the upper bound for the entanglement of distillation $E_{D}(\rho)$.

Proof: Assume that there exists a state $\rho$ such that 
$\tilde{E}(\rho)<a<E_{D}(\rho)$. However, we can asymptotically distill $m E_{D}(\rho)$ numbers of the maximally entangled states from the state $\rho^{\otimes m}$, by definition. Then we choose a pure state such that $E_{p}(|\Psi\rangle\langle\Psi|)=a$. Some of the $m E_{D}(\rho)$ numbers of the maximally entangled states can be asymptotically transformed to $(|\Psi\rangle\langle\Psi|)^{\otimes m}$, since $m E_{D}(\rho)>m a$. Thus, we have done asymptotic transformation $\rho \Rightarrow|\Psi\rangle\langle\Psi|$. By defintion 1, we have $\tilde{E}(\rho) \geq \tilde{E}(|\Psi\rangle\langle\Psi|)$. By the normalization condition, we have $\tilde{E}(|\Psi\rangle\langle\Psi|)=a$. Thus we have $\tilde{E}(\rho)<\tilde{E}(|\Psi\rangle\langle\Psi|)$, which contradicts the above obtained inequality.

The entanglement measures with strong-monotonicity satisfying certain additional conditions in Ref. [8,9] are bounds for the asymptotic measures, namely the entanglement of cost $E_{C}$ and entanglement of distillation $E_{D}$. As we have shown, the entanglement measures with asymptotic weak-monotonicity satisfying an additional normalization condition are also bounds for the asymptotic measures. Due to the existence of the additional conditions, the two types of bounds can be different.

In conclusion, we have proposed entanglement measures with asymptotic weak-monotonicity. The orderings of the entanglement measures $E(\rho)$ with asymptotic weak-monotonicity for the pure states is the same as that of the unique measure of entanglement $E_{p}(\rho)$ for the pure states [24]7]. This fact enabled us to reparameterize entanglement measures with asymptotic weak-monotonicity $E(\rho)$ such that the $E(\rho)$ coincides with $E_{p}(\rho)$ for all pure states. We have shown that normalized entanglement measures with asymptotic weakmonotonicity $\widetilde{E}$ are lower (upper) bound for the entanglement of cost $E_{C}$ (entanglement of distillation $E_{D}$ ).

\section{ACKNOWLEDGMENTS}

We are very grateful to Prof. Hiroshi Imai and Japan Science Technology Cooperation for financial supports. We are also very grateful to Dr. G. Vidal for helpful discussions.

* Corresponding author, Email: wyhwang@qci.jst.go.jp

$\dagger \quad$ Email: keiji@qci.jst.go.jp

[1] A. Einstein, B. Podolsky and N. Rosen, Phys. Rev. 47 (1935) 777; F. Selleri, ed., Quantum Mechanics versus Local Realism. The Einstein-Podolsky-Rosen Paradox (Plenum, New York, 1988).

[2] J.S. Bell, Physics 1 (1964) 195, reprinted in Speakable and Unspeakable in Quantum Mechanics (Cambridge University Press, 1987).

[3] P. Shor, Proc. 35th Ann. Symp. on Found. of Computer Science (IEEE Comp. Soc. Press, Los Alomitos, CA, 1994) 124.
[4] C.H. Bennett, G. Brassard, C. Crepeau, R. Jozsa, A. Peres, and W.K. Wootters, Phys. Rev. Lett. 70 (1993) 1895.

[5] C.H. Bennett, D.P. Divincenzo, J.A. Smolin, and W.K. Wootters, Phys. Rev. A 54 (1996) 3824.

[6] V. Vederal and M.B. Plenio, Phys. Rev. A 57 (1998) 1619.

[7] G. Vidal, J. Mod. Opt. 47 (2000) 355.

[8] M. Horodecki, P. Horodecki, and R. Horodecki, Phys. Rev. Lett. 84 (2000) 2014.

[9] M. J. Donald, M. Horodecki, and O. Rudolph, quant$\mathrm{ph} / 0105017$.

[10] P.M. Hayden, M. Horodecki, and B.M. Terhal, J. Phys. A 34 (2001) 6891.

[11] G. Vidal and J.I. Cirac, Phys. Rev. Lett. 86 (2001) 5803.

[12] G. Vidal, W. Dür, and J. I. Cirac, quant-ph/0112131.

[13] T. Shimono, quant-ph/0203039.

[14] M. Horodecki, P. Horodecki, and R. Horodecki Phys. Rev. Lett. 86, 5844 (2001).

[15] G. Vidal and R.F. Werner, Phys. Rev. A 65, 032314 (2002).

[16] M. Horodecki, Quantum Information and Computation 1 (2001) 3, references therein.

[17] O. Rudolph, Journal of Mathematical Physics 42 (2001) 5306.

[18] A. Uhlmann, Rep. Math. Phys. 9 (1976) 273.

[19] R. Jozsa, J. Mod. Opt. 41 (1994) 2315.

[20] M.A. Nielsen, Phys. Rev. Lett. 83 (1999) 436.

[21] C.H. Bennett, S. Popescu, D. Rohrich, J.A. Smolin, and A.V. Thapliyal, Phys. Rev. A 63 (2001) 012307.

$[22]$ W.Y. Hwang, J. Lee, D. Ahn, and S.W. Hwang, quant$\mathrm{ph} / 0011010$.

[23] C.H. Bennett, H.J. Berstein, S. Popescu, and B. Schmacher, Phys. Rev. A 53 (1996) 2046.

[24] S. Popescu and D. Rorhlich, Phys. Rev. A 56 (1997) 3319.

[25] S. Virmani and M.B. Plenio, Phys. Lett. A 268 (2000) 31. 